\title{
High fidelity transfer of nanometric random textures by UV embossing for thin film solar cells applications
}

\author{
Jordi Escarré*, Karin Söderström, Corsin Battaglia, Franz-Josef Haug, Christophe Ballif \\ Ecole Polytechnique Fédérale de Lausanne (EPFL), Institute of Microengineering (IMT), Photovoltaics and Thin Film Electronics Laboratory, Rue A.-L. Breguet 2, \\ 2000 Neuchâtel, Switzerland
}

\section{A R T I C L E I N F O}

\section{Article history:}

Received 5 July 2010

Received in revised form

27 October 2010

Accepted 13 November 2010

Available online 26 November 2010

Keywords:

Solar cells

Thin film

Plastic substrates

Light trapping

UV imprinting

\begin{abstract}
A B S T R A C T
We investigate the transfer of random nanostructures commonly used in thin film silicon solar cells onto inexpensive substrates, such as glass or flexible polyethylene sheets. Morphological and optical analyses of masters and replicas show the successful transfer of details with sizes much below $1 \mu \mathrm{m}$. These highquality replicas are obtained by UV nano-imprinting, avoiding the use of PDMS as an intermediate mold, which has been identified as being responsible for the lack of resolution found in previous works.
\end{abstract}

(c) 2010 Elsevier B.V. All rights reserved.

\section{Introduction}

Light trapping techniques play an important role in increasing the efficiency of thin film amorphous silicon (a-Si:H) solar cells by improving light absorption without requiring thicker devices. The use of thinner active layers leads to solar cells with better carrier collection and higher stable efficiencies. The most common approach to confine the light relies on natural structures that either the substrate or the contact layers develop under specific deposition conditions during their growth. These rough interfaces cause the light to scatter, increasing its optical path inside the active layer and leading to devices with higher short circuit current. While there is still debate on the type of the ideal roughness, high currents $\left(\sim 17.5 \mathrm{~mA} / \mathrm{cm}^{2}\right)$ have already been obtained in superstrate configuration $(\mathrm{p}-\mathrm{i}-\mathrm{n}) \mathrm{a}-\mathrm{Si}: \mathrm{H}$ devices using the as-grown boron doped zinc oxide ( $\mathrm{ZnO}: \mathrm{B}$ ) pyramidal texture deposited on glass by low pressure chemical vapor deposition (LPCVD) [1], or F-doped $\mathrm{SnO}_{2}$ deposited by atmospheric pressure chemical vapor deposition (AP-CVD) [2]. Likewise, the texture that develops when silver is deposited by sputtering on substrates heated to $\sim 400{ }^{\circ} \mathrm{C}$ is widely used to efficiently scatter light in substrate configuration $(\mathrm{n}-\mathrm{i}-\mathrm{p})$ solar cells, where the substrate transparency is not a requirement [3-5]. However, these kinds of texture are extremely difficult or even impossible to be applied on inexpensive plastic foils due to the low thermal resistance, or the higher dilatation

\footnotetext{
* Corresponding author. Tel.: +4132718 33 79; fax: +41 327183201 .

E-mail address: jordi.escarre@epfl.ch (J. Escarré).
}

coefficient of the plastics compared to glass, which results in the peeling of the layers. Nowadays, the interest in the use of plastics as substrates for thin silicon solar cells is increasing in order to reduce the manufacturing costs by means of roll to roll deposition [6], and because flexible light weight modules offer novel possibilities in building integration. Thus, alternative ways to obtain suitable photovoltaic textures on plastics as polyethylene naphtalate (PEN) have to be found.

Ultraviolet nanoimprint lithography (UV-NIL) and hot embossing lithography (HEL) techniques are possible options to transfer in a controlled way a master texture on top of plastic substrates. Good results concerning resolution and reproducibility have been reported using these techniques $[7,8]$. Note that both techniques require the fabrication and use of an intermediate mold with the negative texture of the original master. Electrochemically deposited nickel and poly-dimethyl siloxane (PDMS) are employed as intermediate molds in HEL and UV-NIL, respectively. Moreover, both techniques are easily scalable to large areas and can be implemented in roll to roll systems [9,10]. UV-NIL avoids possible thermal limitations imposed by the substrate and requires less time and lower pressure ( $\sim 1$ bar) to obtain high quality replicas than hot embossing. Nowadays, UV-NIL is mostly used in the transfer of regular and periodic nanometric structures rather than in the replication of random multiscale textures, which is probably a more challenging task. In a previous work [11] we studied the UVNIL stamping of random textures using PDMS intermediate molds. A lack of resolution for sub-micrometer size features was noticed. Several reports point out the fabrication of the PDMS mold as the limiting factor to high fidelity replication; either its high viscosity 
[12] or its easy deformability [13] seems to be an important drawback for an optimum sub-micron resolution. However, different approaches in the intermediate mold fabrication lead to resolutions around $50 \mathrm{~nm}$, which have been achieved by working with more favorable master textures consisting of periodical arrangements of dots [12] and square shaped pillars [14].

In this work we present results concerning the stamping of random textures by means of UV-NIL using two different types of intermediate molds. The first one consists of the traditional soft undiluted PDMS, while the second one is made from a UV curable sol-gel material that we apply to a flexible substrate in order to facilitate demolding. A rigorous comparison was carried out for both stamping methods, identifying the use of PDMS as problematic in order to achieve high fidelity replicas of random nanometric textures.

\section{Experimental}

Glass (0.5 mm thick, Schott AF45) with a size of $4 \times 4 \mathrm{~cm}^{2}$ was used as a substrate in the replication process. Two different types ( $\mathrm{A}$ and $\mathrm{B}$ ) of as-grown textured $\mathrm{ZnO}$ : $\mathrm{B}$ by LPCVD were used as masters in the replication tests. Both textures exhibit a similar pyramidal shape but with different feature sizes. Type A presents smaller and shallower pyramids than type B. All the steps followed to transfer the master textures on glass are shown in Fig. 1. Two

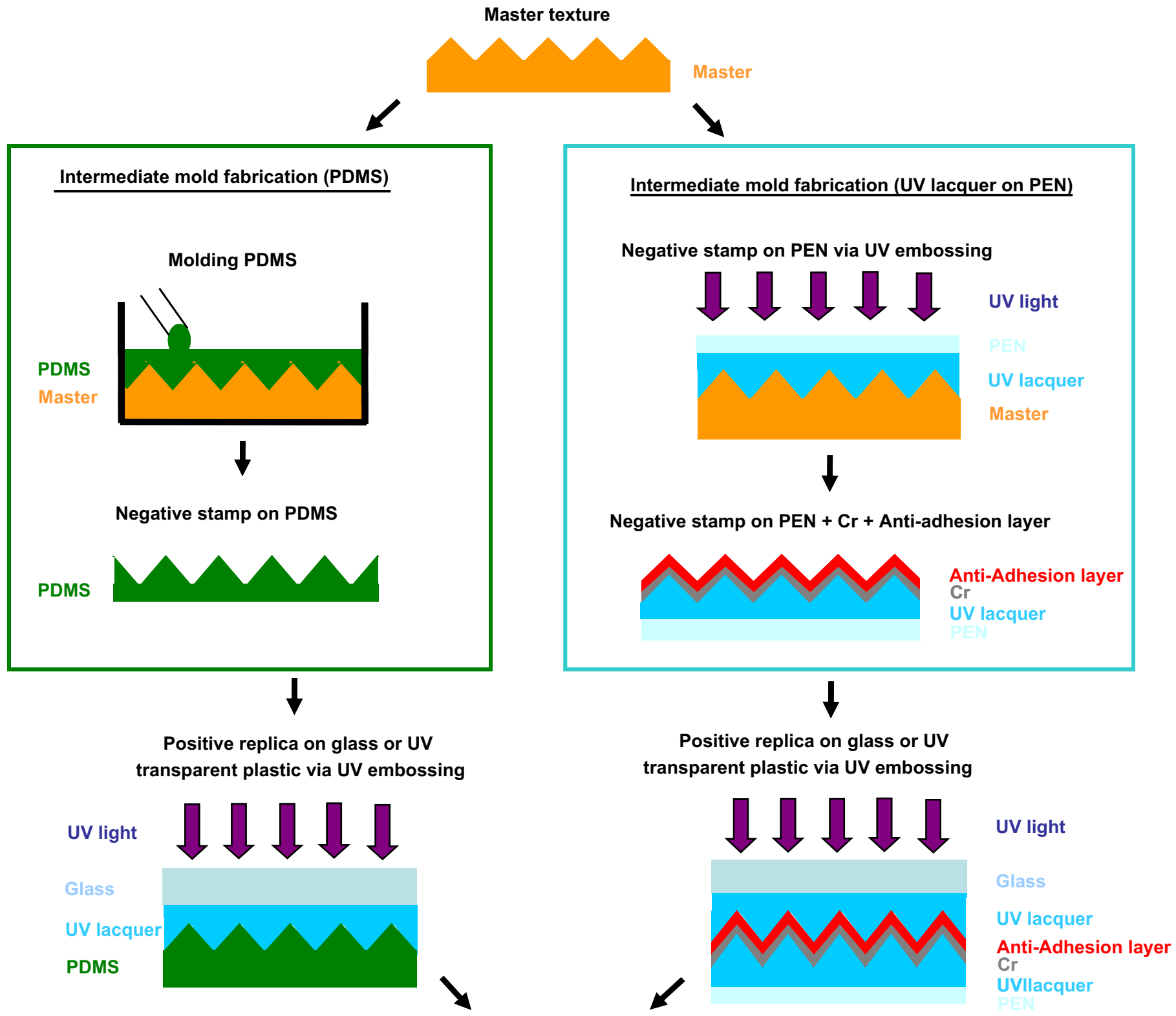

Positive replica on glass or UV transparent plastic

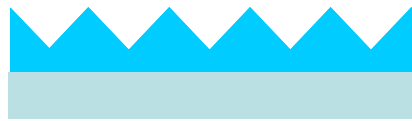

UV lacquer

Glass

Fig. 1. Replication process steps followed for the transfer of the master texture on glass by UV embossing. 
different kinds of intermediate mold were fabricated from the masters. The first one was obtained in PDMS (Sylgard 184, Dow Corning). PDMS was prepared with a ratio 10:1 between the base and the curing agent, degassed in vacuum, and cured at $46{ }^{\circ} \mathrm{C}$ for $12 \mathrm{~h}$. The second one was obtained by UV-NIL embossing of the master texture into a commercially available UV sensitive sol-gel lacquer layer that was spin coated onto a flexible PEN sheet $(0.125 \mathrm{~mm}$ thick, Goodfellow). After curing and demolding from the master, we coated the flexible stamp with a thin $\mathrm{Cr}$ layer $(\sim 40 \mathrm{~nm})$ and an antiadhesion monolayer of fluoroalkylsilane (trichloro(tridecafluorooctyl)silane; United Chemical Technologies) [15].

UV-NIL stamping was carried out in a 'homemade' system by applying homogenous pressures around 1 bar. The stamping steps were realized under vacuum to avoid gas bubble formation during the contact of mold and UV lacquer. The photocurable lacquer was then exposed to a moderate intensity $\left(1.4 \mathrm{~mW} / \mathrm{cm}^{2}\right)$ of UV light $(\sim 365 \mathrm{~nm}$ ) coming through the substrate. PEN substrates require longer exposure ( $\sim 20 \mathrm{~min})$ than glass $(\sim 1 \mathrm{~min})$ because of their lower UV transmission.

Replication fidelity was studied morphologically by means of scanning electronic microscopy (SEM) and atomic force microscopy (AFM) after the deposition of $\sim 120 \mathrm{~nm}$ of silver on both masters and replicas. As in previous work [11], the root mean square roughness $\left(\sigma_{\mathrm{RMS}}\right)$, the correlation length $(L)$, the mean angle $(\langle\alpha\rangle)$ and the power spectral density (PSD) were extracted to compare the master and replica structures in a quantitative way. $\sigma_{\text {RMS }}$ gives information about the vertical size of the roughness whereas $L$ represents the lateral size distribution of the surface texture. Note that $\sigma_{\mathrm{RMS}}$ and $L$ represent the commonly used approximation of the surface height distribution and the autocorrelation function, respectively, by Gaussian distributions [16]. $L$ was calculated as the radius, where the auto-correlation peak drops to $1 / \mathrm{e}$ of its maximum value, assuming circular shape. The mean angle is the average angle between the surface normal and the local inclination evaluated for every point of the AFM image. $P S D$ is obtained from the fast Fourier transform of the images, and indicates the weight of the different sinusoidal components whose linear combination corresponds to the analyzed surface.

The haze ratio and the angle resolved scattering curve were measured in reflection to optically compare master and replica surfaces previously covered with a sputtered silver film $(\sim 200 \mathrm{~nm}$; Univex 50B, Leybold, deposited at room temperature). The haze ratio is calculated as the fraction of scattered light to total reflected light (specular + scattered). Both magnitudes, specular and scattered light, were measured in the spectral range from 350 to $2000 \mathrm{~nm}$ using a spectrophotometer with an integrating sphere (Lamda 900, Perkin Elmer). Angle resolved scattering measurements were carried out at a wavelength of $543 \mathrm{~nm}$.

For an assessment of the suitability in devices, single junction microcrystalline solar cells in $\mathrm{p}-\mathrm{i}-\mathrm{n}$ configuration with an intrinsic layer thickness of $1.2 \mu \mathrm{m}$ were grown on type A samples. In the replicas, the front electrical contact was achieved by depositing a thin indium tin oxide (ITO) layer with a thickness around $100 \mathrm{~nm}$. More details concerning deposition of $\mathrm{p}-\mathrm{i}-\mathrm{n}$ cells on UV replicas can be found elsewhere [17]. The solar cells were characterized by measuring the external quantum efficiency (EQE). The short circuit current density $\left(J_{\mathrm{sc}}\right)$ of the devices was calculated by integrating over the convolution of EQE and AM $1.5 \mathrm{~g}$ spectra.

\section{Results and discussion}

Firstly, differences in replica fidelity were studied using type A texture as a master. The SEM images shown in Fig. 2 compare the original surface of the master and the subsequent UV-NIL embossed surfaces on glass, using the two different intermediate
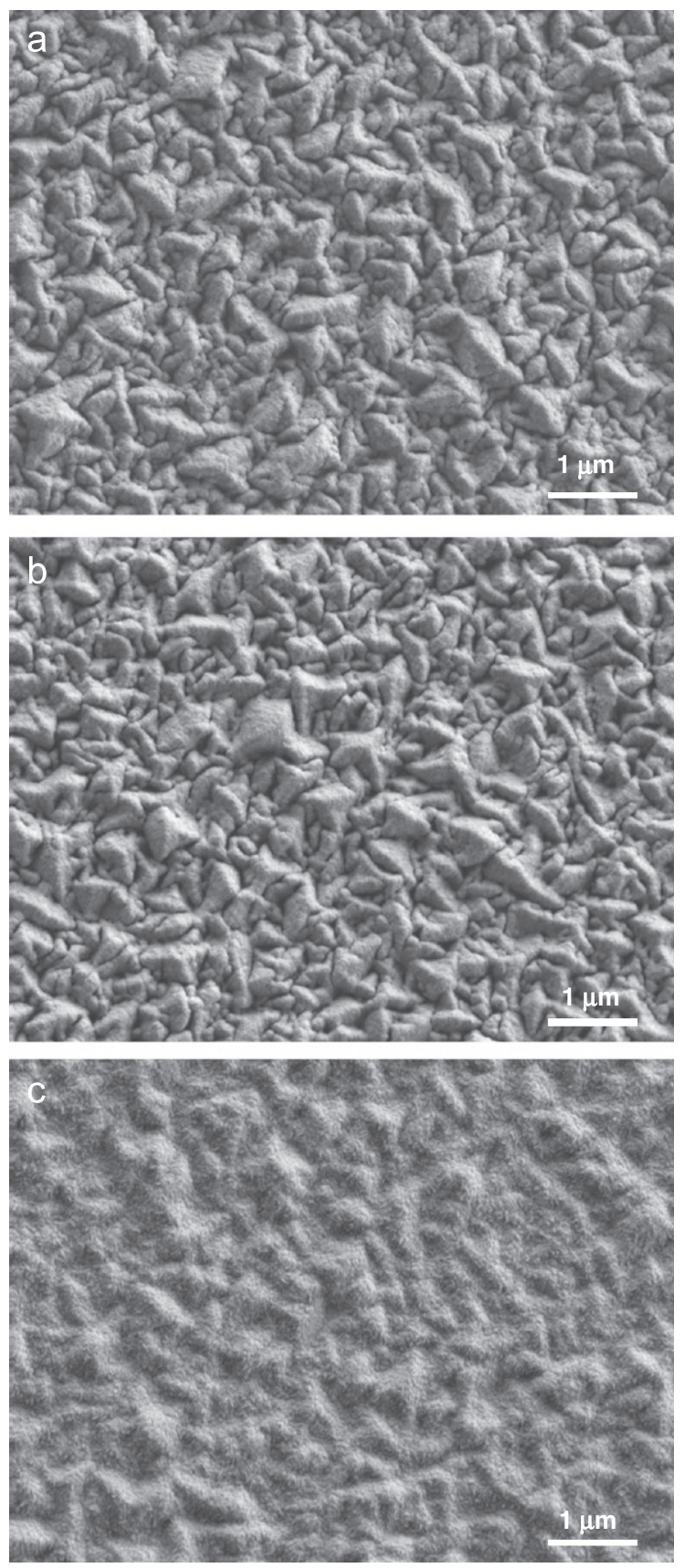

Fig. 2. SEM images of (a) type A master and UV imprinted replicas (b) without PDMS and (c) using PDMS to fabricate the intermediate mold.

molds. The master in Fig. 2(a) contains the typical pyramidal texture of the $\mathrm{ZnO}$ grown by LPCVD, with a mean separation between peaks of $0.7 \mu \mathrm{m}$. Stamping the UV lacquer on glass using the intermediate UV-NIL mold produces a surface with feature shapes similar to the original ones (Fig. 2(b)). The image of the surface of the replica, stamped with the intermediate PDMS mold (Fig. 2(c)), reveals an imprinting with smoother features.

For better comparison, additional information extracted from AFM images (size $10 \times 10 \mu \mathrm{m}^{2}$ ) of master and replicas is summarized in Fig. 3. Height histograms (Fig. 3(a)) reveal a narrow distribution for the replica stamped with PDMS. A lower $\sigma_{\mathrm{RMS}}$ value of $55.8 \mathrm{~nm}$ was estimated for the replica compared with that of $69.8 \mathrm{~nm}$ for the master. Besides, correlation lengths values of 201 and $165 \mathrm{~nm}$ were calculated for replica and the master, respectively, using the transversal section of the auto-correlation function 
a

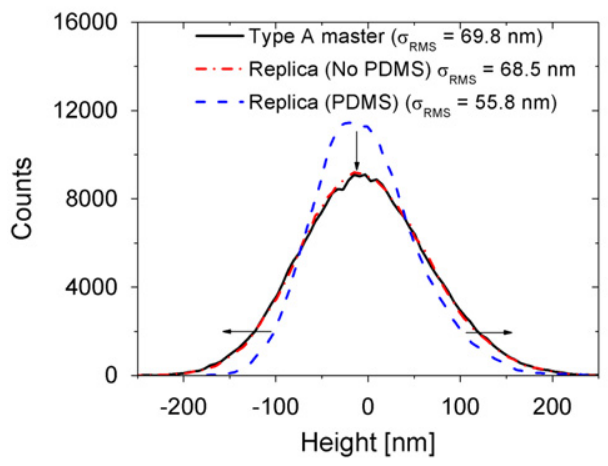

C

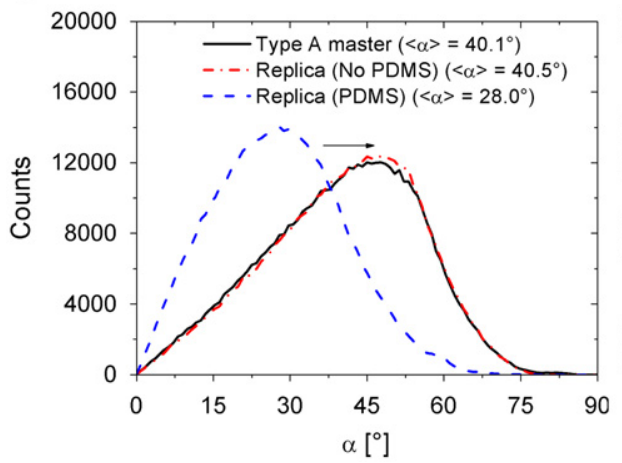

b

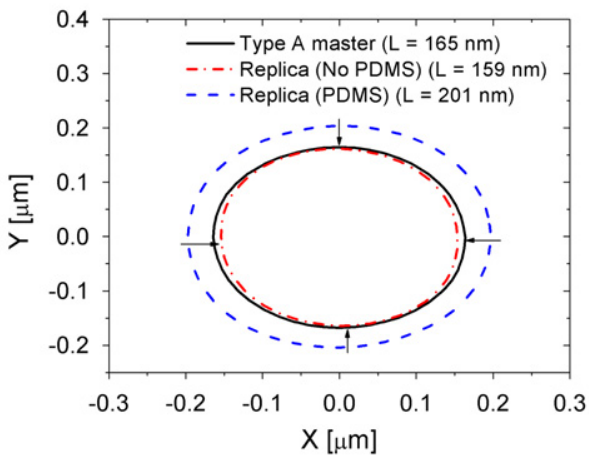

d

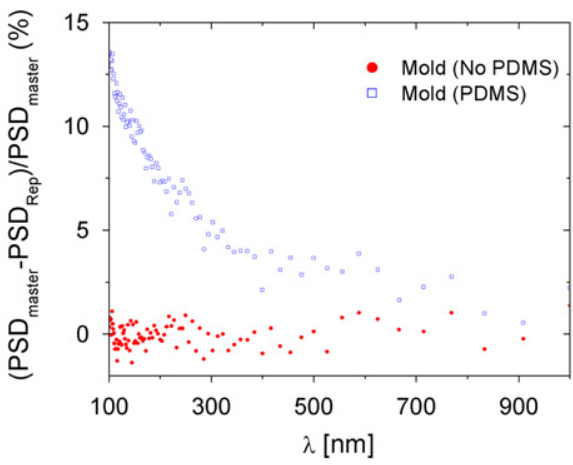

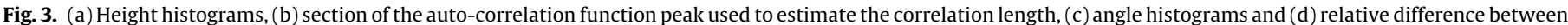
the PSD of master and replicas calculated from the AFM images of the samples shown in Fig. 2.
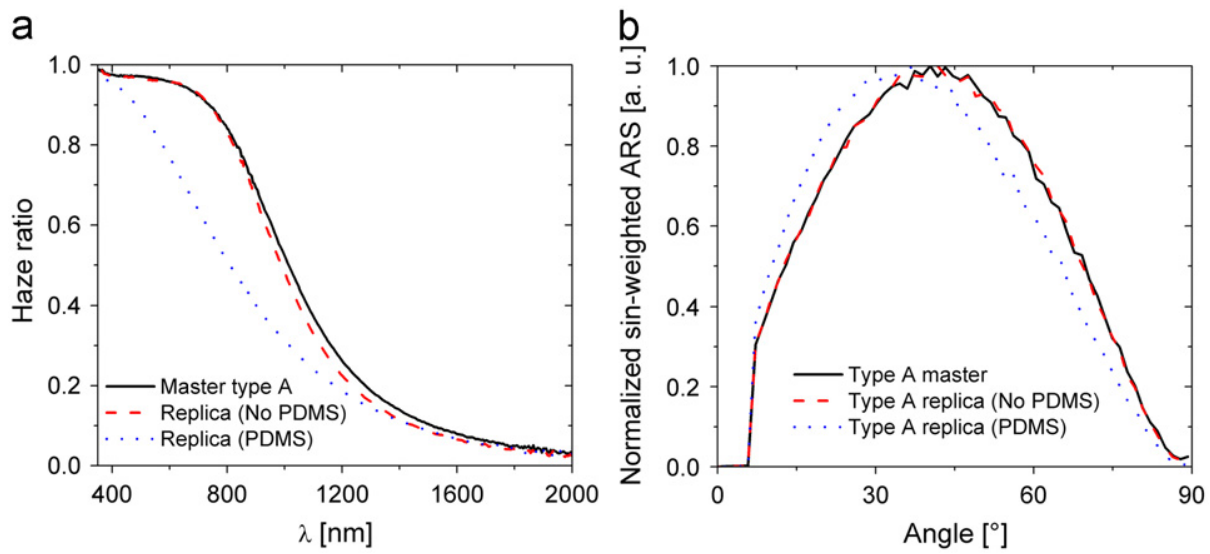

Fig. 4. (a) Haze ratio measurements and (b) normalized sin-weighted ARS curves measured for type A master and replicas.

peak shown in Fig. 3(b). The longer correlation length estimated for the replica suggests a texture consisting of larger lateral features but is in fact due to the loss of fine surface details. For the replica, the combination of both shallow and larger lateral features gives a texture with a lower aspect ratio. This fact is noticeable in the angular histograms (Fig. 3(c)), where the master exhibits slopes whose mean local inclination is around $12^{\circ}$ steeper than that of the replica. The smoothing of the replica surface is related to a nonaccurate imprinting of sub-micron features as can be seen in Fig. 3(d). The relative difference between both master and replica PSD curves is seen to increase towards $13 \%$ as the length of the probed features is decreased from 700 to $100 \mathrm{~nm}$. These results confirm the difficulty in obtaining high quality replicas of type A texture by UV-NIL using an intermediate mold made from PDMS. The same kind of morphological comparison was done for the master and the replica obtained using the intermediate UV-NIL mold fabricated on PEN (Fig. 3). No significant differences can be found between both surfaces, confirming that avoiding the use of PDMS high-fidelity replicas of photovoltaic, multiscale random structures can be obtained on glass or on PEN by UV-NIL imprinting.

Optical measurements were also carried out in order to better compare the surface of type A master and replicas. Optical measurements are helpful tools to thoroughly compare master and replicas due to their high sensitivity to the morphology of the samples. In Fig. 4(a), the haze ratio measured for the replica stamped without PDMS follows closely the values measured for the master because of its similar texture. The replica stamped using PDMS presents a haze ratio curve with different shape, scattering less amount of light in the whole spectral range due to its lower 
roughness. Besides the ratio of light scattered for each sample, the angular distribution in which light is scattered is also very important to compare the rough surface of the masters and the replicas. The normalized sin-weighted angle resolved scattering curve measured for type A samples is shown in Fig. 4(b). The master and the replica stamped without PDMS scatter the maximum amount of light at the same angle $\left(\sim 42^{\circ}\right)$ and no difference can be observed in the way light is angularly scattered for both samples. On the other hand, the replica stamped with PDMS mostly scatters light at a lower angle $\left(\sim 37^{\circ}\right)$. The shift of the curves to lower angles is clearly a consequence of the smoother slopes present in its surface compared with the ones in the master.

Finally, microcrystalline $\mathrm{p}-\mathrm{i}-\mathrm{n}$ solar cells were co-deposited on type A master and replicas. Fig. 5 shows the EQE curves measured for the cells. At short wavelengths (400-550 nm), the differences between curves are typically related to the substrate texture, which leads to different thicknesses and nucleation of the microcrystalline p-layer, and different reflection losses. Generally, in codeposited solar cells, better responses at short wavelengths and lower open circuit voltages are observed when working with rough substrates. The cells deposited on the master and replica stamped without PDMS present an equivalent behavior at short wavelengths, while the response of the replica stamped with PDMS is significantly lower. The same trend is observed at large wavelengths (550-1100 nm). In this region, the absorbance of the silicon is low and the response of the cell is mainly controlled by the texture of the substrate. The replica stamped using PDMS shows significantly lower response compared to the master. Only small differences can be observed between the master and the replica stamped avoiding PDMS. The slightly lower EQE in the replica can be explained by the higher absorption of the ITO front contact with respect to the $\mathrm{ZnO}$ one of the master [17]. This result confirms that high quality replicas can be achieved using this new replication method.

Similar experiments were carried out starting from a rougher master texture. The textured surface of type B master is shown in the SEM images of Fig. 6(a). This texture presents the same pyramidal shape as that of type A master but with larger sizes, exhibiting a mean separation of $1.8 \mu \mathrm{m}$ between peaks. Finer details are observed on some of the planes of the pyramids in forms of terracing, which are related to the growth of ZnO by LPCVD (see circle in Fig. 6(a)). Type B master was used to fabricate the intermediate mold without using PDMS. Based on the observations using type A texture, we did not study PDMS molds for type B

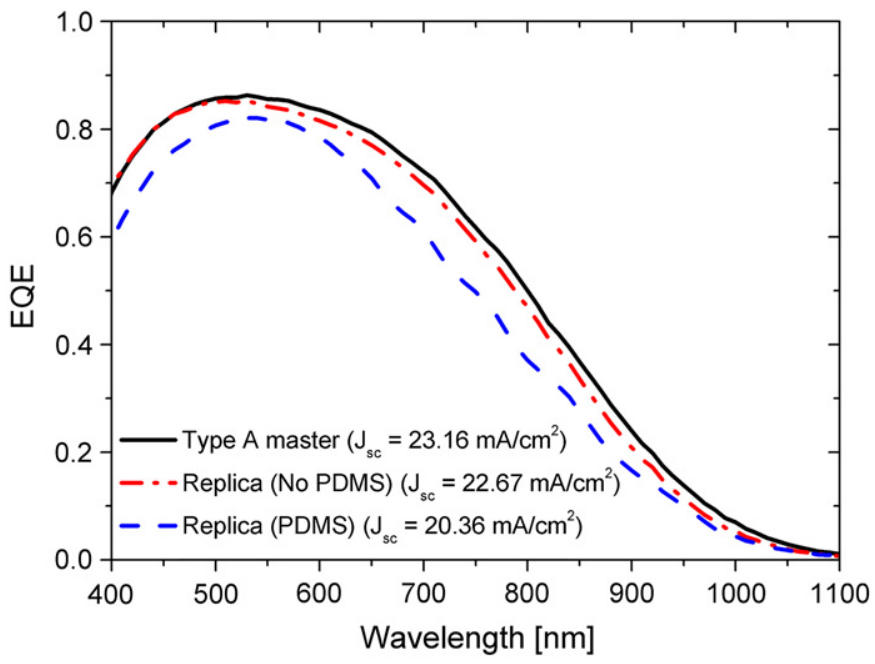

Fig. 5. External quantum efficiency curves of co-deposited microcrystalline silicon solar cells growth on type A master and replicas.
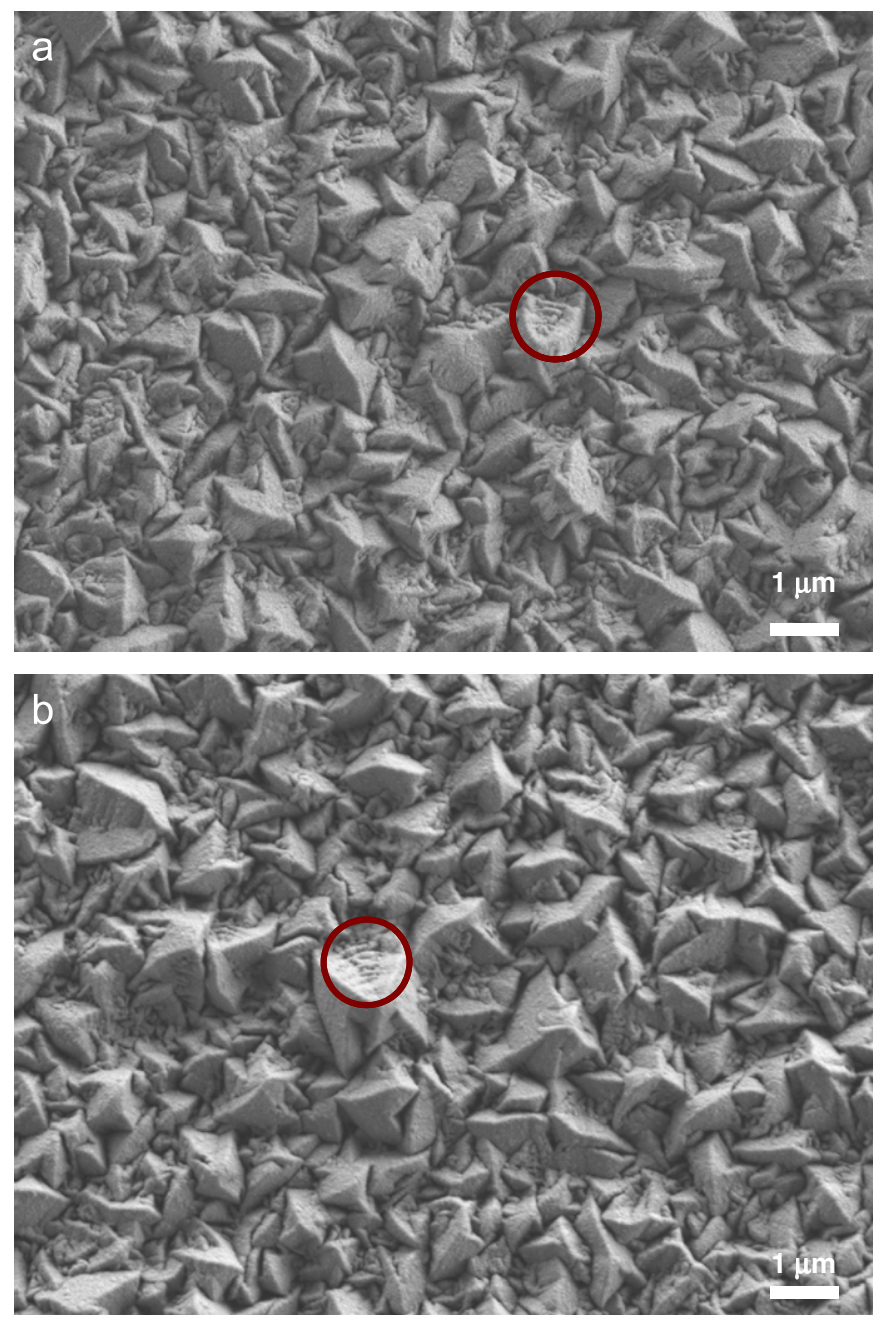

Fig. 6. (a) SEM images of type B master and (b) UV imprinted replica by avoiding PDMS to fabricate the intermediate mold.

texture. However in the previous study made on PDMS replicas [11] larger losses during replication were observed for a $\mathrm{ZnO}$ grown by LPCVD of larger feature size than type A master. The replica is shown in Fig. 6(b). The replicated pyramids are very similar to the ones of the master in size and shape, and there is even evidence of the replication of the terracing observed in the master (circle in Fig. 6(b)). A morphological and optical comparison between master and replica is shown in Fig. 7. Angular histograms calculated from AFM images (size $10 \times 10 \mu \mathrm{m}^{2}$ ) and normalized sin-weighted angular resolved scattering curves are shown in Fig. 7(a) and (b), respectively, for master and replica. The agreement between master and replica is excellent for both measurements, confirming that the UV-mold was able to imprint the rougher type B master to its full extent.

These results confirm excellent transfer of the random roughness of the ZnO:B deposited by LPCVD when using a UV-NIL intermediate mold fabricated on PEN; both the master as well as the replicated textures are equivalent in terms of their morphology and their optical properties. In future work, new approaches have to be tested to solve the drawback of intermediate mold opacity. UV transparency would increase the flexibility of the process by enabling curing of the UV photo sensible lacquer through the mold, making the imprinting process independent of the type of substrate used. Currently, PDMS is UV transparent but its use in intermediate molds leads to replicas with smoother textures. For solar cell applications, the smoothing of these particular master 
a

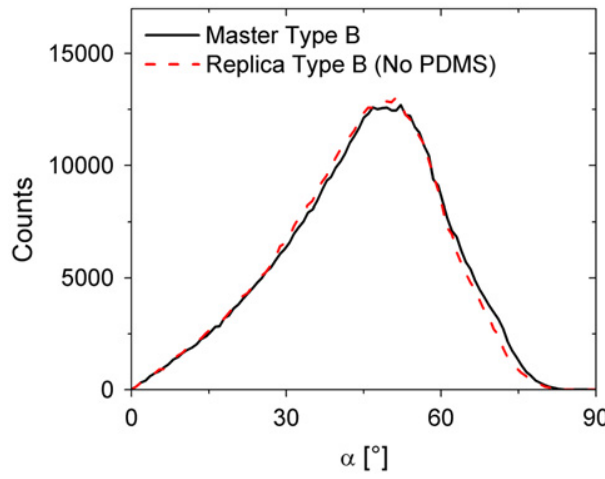

b

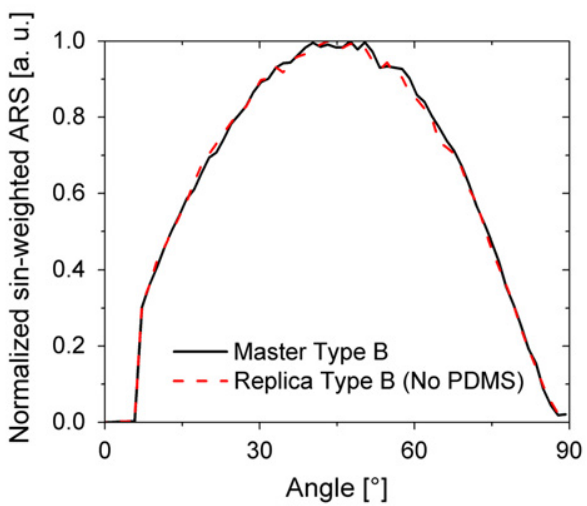

Fig. 7. (a) Angle histograms and (b) normalized sin-weighted ARS curves measured for type B master and replica.

textures has been demonstrated to be beneficial in some cases $[11,18]$. However, note that beneficial or detrimental effects of replication losses cannot be predicted in general as they depend on the original textures and configuration of the devices.

Another remarkable point is that the UV curable lacquer on glass can also be used in solar cells in $\mathrm{p}-\mathrm{i}-\mathrm{n}$ configuration because of its excellent transparency and good stability [11]. No significant differences can be observed in the transmission of the light between a glass and a glass coated with the UV curable lacquer in the whole spectral range comprised between 400 and $1100 \mathrm{~nm}$, even after $2 \mathrm{~h}$ of $\mathrm{UV}$ illumination at $60^{\circ} \mathrm{C}$. These facts make feasible the use of either originally non-transparent or inverted textures in this kind of configuration, adding a new tool to better understand the effect of the roughness on the optical, electrical and structural properties of these devices.

\section{Conclusions}

We presented a procedure for high fidelity replication of the asgrown textured $\mathrm{ZnO}$ : B deposited by LPCVD, which is commonly used in thin film silicon technology. The use of PDMS as an intermediate mold has been found to be problematic for imprinting random features with sizes below $1 \mu \mathrm{m}$ with high accuracy. The intermediate molds obtained by UV-NIL on PEN have been demonstrated to work properly. These results open up exciting opportunities for better understanding the effect of the texture in solar cell performance, giving the possibility to try inverted textures or typical n-i-p textures in $\mathrm{p}-\mathrm{i}-\mathrm{n}$ solar cells, and vice versa.

\section{Acknowledgments}

The authors gratefully acknowledge support by the Swiss Federal Office for Energy (OFEN) under Contract 101191, the Swiss Commission for Technology and Innovation (CTI) within Project no. 8809.2 and the Swiss National Science Foundation under Grant 200021-125177.

\section{References}

[1] J. Meier, J. Spitznagel, U. Kroll, C. Bucher, S. Fä̈, T. Moriarty, A. Shah, Potential of amorphous and microcrystalline silicon solar cells, Thin Solid Films 451-452 (2004) 518-524.

[2] M. Kambe, M. Fukawa, N. Taneda, Y. Yoshikawa, K. Sato, K. Ohki, S. Hiza, A. Yamada, M. Konagai, Improvement of light-trapping effect on microcrystalline silicon solar cells by using high haze transparent conductive oxide films, in: Proceedings of the Third World Conference Photovoltaic Energy Conversion (2003) 1812-1815.

[3] V. Terrazzoni-Daudrix, J. Guillet, F. Freitas, A. Shah, C. Ballif, P. Winkler, M. Ferreloc, S. Benagli, X. Niquille, D. Fischer, R. Morf, Characterisation of rough reflecting substrates incorporated into thin-film silicon solar cells, Progress in Photovoltaics: Research and Applications 14 (2006) 485-498.

[4] S. Guha, J. Yang, Progress in amorphous and nanocrystalline silicon solar cells, Journal of Non-Crystalline Solids 352 (2006) 1917-1921.

[5] H.B.T. Li, K.H.M. van der Werf, J.K.R. Rath, R.E.I. Schropp, Hot wire CVD deposition of nanocrystalline silicon solar cells on rough substrates, Thin Solid Films 517 (2009) 3476-3480.

[6] T. Söderström, F.-J. Haug, V. Terrazzoni-Daudrix, C. Ballif, Optimization of amorphous silicon thin film solar cells for flexible photovoltaics. Journal of Applied Physics 103 (2008) 114509-1-114509-8.

[7] S.Y. Chou, P.R. Krauss, P.J. Rennstrom, Imprint of sub-25 nm vias and trenches in polymers, Applied Phyics Letters 67 (1995) 3114-3116.

[8] B. Vratzov, A. Fuchs, M. Lemme, W. Henschel, H. Kurz, Large scale ultravioletbased nanoimprint lithography, Journal of Vacuum Science and Technology B 21 (2003) 2760-2764

[9] T. Mäkelä, T. Haatainen, P. Majander, J. Ahopelto, V. Lambertini, Continuous double-sided roll-to-roll imprinting of polymer film, Japanese Journal of Applied Physics 47 (6) (2008) 5142-5144.

[10] S.H. Ahn, J.-S. Kim, L.J. Guo, Bilayer metal wire-grid polarizer fabricated by rollto-roll nanoimprint lithography on flexible plastic substrate, Journal of Vacuum Science and Technology B 25 (6) (2007) 2388-2391.

[11] K. Söderström, J. Escarré, O. Cubero, F.-J. Haug, S. Perregaux, C. Ballif, UV-nanoimprint lithography technique for the replication of back reflectors for $n-\mathrm{i}-\mathrm{p}$ thin film silicon solar cells, Progress in Photovoltaics: Research and Applications (2010) n/a. doi: 10.1002/pip.1003.

[12] N. Koo, M. Bender, U. Plachetka, A. Fuchs, T. Wahlbrink, J. Bolten, H. Kurz, Improved mold fabrication for the definition of high quality nanopatterns by soft UV-nanoimprint lithography using diluted PDMS, Microelectronic Engineering 84 (2007) 904-908.

13] S. Gilles, M. Meier, M. Prömpers, A. van der Hart, C. Kügeler, A. Offenhäusser D. Mayer, UV nanoimprint lithography with rigid polymer molds, Microelectronic Engineering 86 (2009) 661-664.

[14] M. Mühlberger, I. Bergmair, A. Klukowska, A. Kolander, H. Leichtfried E. Platzgummer, H. Loeschner, Ch. Ebm, G. Grützner, R. Schöftner, UV-NIL with working stamps made from Ormostamp, Microelectronic Engineering 86 (2009) 691-693.

[15] C.A. Mills, J. Escarré, E. Engel, E. Martinez, A. Errachid, J. Bertomeu, J. Andreu, J.A. Planell, J. Samitier, Micro- and nanostructuring of poly(ethylene-2,6naphtalate) surfaces, for biomedical applications, using polymer replication techniques, Nanotechnology 16 (2005) 369-375.

[16] H. Davies, The reflection of electromagnetic waves from a rough surface, in: Proceedings of the American Academy for Jewish research (1954) 209-214.

[17] C. Battaglia, K. Söderström, J. Escarré, F.-J. Haug, D. Dominé, P. Cuony, G. Mathieu Boccard, C. Bugnon, M. Denizot, A. Despeisse, Feltrin, C. Ballif, Efficient light management scheme for thin film silicon solar cells via transparent random nanostructures fabricated by nanoimprinting, Applied Physics Letters 96 (2010) 213504-1-213504-3.

[18] J. Bailat, D. Dominé, R. Schlüchter, J. Steinhauser, S. Faÿ, F. Freitas, C. Bücher, L. Feitknecht, X. Niquille, T. Tscharner, A. Shah, C. Ballif, High-efficiency p-i-n microcrystalline and micromorph thin film silicon solar cells deposited on LPCVD ZnO coated glass substrates, in: Proceedings of the Fourth WCPEC Conference (2006) 1533-1536. 